\title{
Eloy Benito Ruano
}

juan lgnacio Ruiz de la Peña Solar

Universidad de Oviedo 

Mis compañeros del Departamento de Historia Medieval de la Universidad Nacional de Educación a Distancia, con ocasión del merecido homenaje que dedican al Profesor Benito Ruano al cumplirse el término administrativo de su docencia activa en aquel centro, me honran con el encargo de hacer la semblanza del querido maestro.

A su lado, durante largos años de estrecha convivencia académica, se consolidaron mi formación científica y mi vocación histórica. $Y$ al final de su dilatada etapa ovetense, en 1981, asumí la responsabilidad de continuar la obra que él había iniciado, en 1964, en la Universidad de Oviedo. Me honro en proclamarme discípulo directo del Profesor Benito Ruano, deudor de su magisterio generoso, colaborador en muchas empresas comunes. $Y$ me honro, sobre todo, con la amistad entrañable de quien ha sido y es para la Universidad española, para la comunidad científica del medievalismo hispano y para los beneficiarios de sus enseñanzas, espejo de virtudes y modelo de dedicación al oficio más noble que es dado ejercer al hombre de tejas abajo: el de quien hace de su vida una dádiva permanente que es, y lo expreso con palabras de $\mathrm{Ma}$ rañón, "el término consciente de una entrega, sin plazos y sin réditos, cuya generosidad no se puede medir", el oficio que encierra una hermosa palabra -maestro- tan grata y evocadora para quienes hemos tenido el privilegio de poder apreciar en nuestro propio espíritu todo su hondísimo significado.

Nace Eloy Benito Ruano en Madrid, el 1 de diciembre (festividad de San Eloy, patrono de los orfebres) de 1921, en el seno de una familia enraizada en las tierras de Toledo, en Olías del Rey. Siempre a lo largo de su vida, se hará presente en la obra del Profesor Benito Ruano una 
querencia especial por esa tierra toledana, escenario de no pocos de sus trabajos históricos.

En Madrid transcurren su infancia y adolescencia, truncada ésta brutalmente por el drama de la guerra, que el joven y ya aventajado estudiante de bachillerato pasa con su familia en la ciudad cercada, alternando la presencia en las aulas con el riesgo bélico de los servicios en la sanidad militar. Tampoco son fáciles los años de la postguerra inmediata: convalidación de los estudios cursados en el Madrid sitiado, duro castigo para el vencido, aunque sea todavía casi un niño, y búsqueda de un trabajo que contribuya al levantamiento de las cargas de un hogar modesto y le permita realizar su gran ilusión: acceder a una carrera universitaria.

Simultaneándolos con el trabajo, Eloy inicia los estudios de Filosofía y Letras en la Universidad de La Laguna, donde le habian llevado los avatares de su destino administrativo, retornando pronto a Madrid, en cuya Universidad Central se licenciaria, en la especialidad de Historia, en 1948, con la calificación de Premio Extraordinario.

De su estancia en Canarias conservaria siempre un indeclinable amor por aquellas tierras isleñas, por sus gentes y por su historia, objeto de los primeros trabajos en los que se perfila ya una clara vocación investigadora. Las Canarias serán luego tema recurrente en la densa bibliografía del Profesor Benito Ruano. De aquellos años guardaría también el recuerdo entrañable del magisterio de Serra Rafols, compartido después en Madrid con otros ilustres historiadores a los que siempre ha rendido el tributo de admiración y de afecto que es patrimonio de las almas nobles: Montero Díaz, Antonio de la Torre, Rumeu de Armas...

Bajo la dirección de estos maestros inicia Benito Ruano su carrera docente en la Facultad de Filosofía y Letras de la Universidad Central de Madrid, ocupando sucesivamente las plazas de Profesor Ayudante (de 1948 a 1958) y Profesor Adjunto de Historia General de España, cuya Cátedra regentaba desde 1949, el Profesor Rumeu de Armas.

Los magros estipendios que devengaba entonces el desempeño de esos cargos académicos obligan a simultanear el ejercicio de la docencia universitaria con otras actividades más remunerativas que permitiesen llegar al menos al umbral de la subsistencia. Eloy Benito continuaría pues, en esos años, al servicio de la Administración Pública, compatibilizando el trabajo extramuros de la Universidad con la actividad docente. 
La obtención de pensiones y becas, tan raras en aquella época, le permiten completar su formación y desarrollar una actividad investigadora en centros y archivos nacionales y extranjeros muy pronto rentabilizada en numerosos y sólidos trabajos.

El primero de ellos, el inevitable banco de pruebas de todo investigador con vocación universitaria, es su tesis doctoral, que defiende en la Universidad Central en 1956 obteniendo la calificación de Premio Extraordinario, y que materializaría años después -tampoco era fácil publicar entonces- en un magnífico libro: Toledo en el siglo xV. Vida política; esta obra, que obtendría el premio "Raimundo Lulio" en 1960 y que se publicaría dos años después bajo los auspicios del Congreso Superior de Investigaciones Científicas, sitúa ya a su autor en un lugar de vanguardia entre la brillante generación de medievalistas españoles de la década de los sesenta. Buena parte de cuantos hoy formamos en las filas de la gran familia de nuestro medievalismo somos deudores inmediatos del magisterio y de los aires de renovación científica que introdujeron en la Universidad española aquellos queridos compañeros, cuyo alejamiento gradual de ella, acelerado en los últimos años por una lamentable política de anticipación del término de la docencia activa, está dejando una cierta sensación de orfandad académica en quienes hace poco más de dos décadas eramos todavía sus discípulos en las aulas.

Un repaso al currículum y a la bibliografía del Profesor Benito Ruano en esa primera etapa que convencionalmente llamamos de formación y que en este caso debiera calificarse con más propiedad de antesala o espera en el acceso a la consagración académica de la Cátedra -entonces la alternativa era Cátedra o nada-, puede constituir todo un ejemplar ejercicio de reflexión sobre lo que significaba el cursus honorum de quienes en aquella dura época tenían arrestos para responder a la vocación universitaria.

Un joven profesor de hoy difícilmente puede imaginar los sacrificios, estrecheces y dificultades de todo tipo que entonces suponían los inicios de una carrera docente universitaria. Por simples razones de proximidad generacional estamos en situación de comprenderlo y valorarlo mejor quienes conocimos de alumnos la que el Profesor Gibert calificaba en cierta ocasión de "universidad sin despachos" y, tentados también por la llamada de la vocación docente e investigadora, escuchábamos en las aulas, como escuche yo de boca de un venerable profesor, fórmulas 
disuasorias del tenor siguiente: «quien quiera dedicarse a la Universidad, se 10 advierto a Vds., que se prepare a hacer los tres votos: pobreza, castidad y obediencia".

Quizá cargaba las tintas aquel anciano y sabio catedrático - don Ramón Prieto Bances- que contribuyó con otros inolvidables maestros a despertar en mi una decidida afición por la historia. Pero por mucho que se tratase de edulcorar la situación, quien la vivia entonces, como la vivió y padeció el Profesor Benito Ruano y tantos otros compañeros de su generación, merecería un premio especial a la constancia vocacional $y$, sobre todo a la esperanza en un futuro profesional velado casi siempre por sombríos nubarrones.

Pero dejemos este tipo de reflexiones para ocasión más oportuna y volvamos ahora al encuentro del querido maestro en aquellos años de paciente espera académica.

Aludíamos antes a la obtención por el Profesor Benito Ruano en esta etapa de diversas pensiones y becas que le permitirán cimentar una sólida fomación de historiador, al tiempo que suponen el reconocimiento de sus sobresalientes méritos en el campo de la investigación y de la docencia. Entre otros centros e instituciones nacionales y extranjeras que le distínguen con sus ayudas para seguir cursos de especialización y facilitar sus trabajos, brindándole el acceso a los principales archivos y bibliotecas europeas y la estrecha relación con prestigiosos maestros propios y foráneos figuran la Universidad Internacional "Menéndez y Pelayo" (1949), la Università per Stranieri de Perugia (1950), la Escuela de Estudios Medievales de Madrid (CSIC, 1951-19.55), la Dirección General de Archivos y Bibliotecas (1953), la Dirección General de Relaciones Culturales del Gobierno Francés (París 1953), el Museo Británico (1955), el Instituto Italiano de Madrid (1958), La Fundación «Juan March» (1962), etc., etc.

Por otra parte, el Colegio Oficial de Doctores y Licenciados en Ciencias y en Letras del Distrito Universitario de Madrid, distinguía en 1956 a Eloy Benito Ruano con el premio anual reservado a los colegiados más destacados.

También tempranamente se vincula el Consejo Superior de Investigaciones Científicas, del que figurará como Colaborador desde 1956, haciéndose cargo dos años después de la secretaría de la revista «Hispania», desde la que desplegó una gestión de cuya constancia y 
eficacia queda fiel reflejo en las páginas de la prestigiosa publicación periódica del CSIC.

En el denso registro de títulos de la bibliografía del Profesor Benito Ruano que cubre esa primera etapa de docente e investigador comprendida entre los años 1949 y 1964, encontramos ya una serie de trabajos que son claros indicadores de los principales polos de atracción que centrarán en el futuro quizá la mayor y mejor parte de la amplia obra historiográfica del Maestro:

a) Investigaciones en marcos regionales: en este primer bloque temático se inscriben sus publicaciones pioneras sobre historia canaria, tributo, como ya quedó apuntado, a los gratos años laguneros de estudiante; y la dedicación, a los temas de historia medieval toledana, que culmina en su magna obra sobre Toledo en el siglo xV.

b) La historia biográfica, matizada por una fuerte coloración literaria, objeto también de una temprana y mantenida atención cuya clave explicativa habría, quizá, que buscarla en la precoz y nunca abandonada sensibilidad poética del Profesor Benito Ruano que descubría públicamente el profesor Rumeu de Armas en la sentida semblanza que le dedicaba en el acto de su solemne recepción en la Academia de la Historia, en mayo del pasado año. En esta línea se sitúa, como estudio pionero, el precioso libro sobre Los Infantes de Aragón, publicado por la Escuela de Estudios Medievales del CSIC en 1952.

c) Las investigaciones sobre Ordenes militares, Reconquista y Cruzada, ámbito historiográfico al que se adscriben varios artículos, algunos muy extensos y de proyección extrapeninsular, en parte reunidos, con otros posteriores, en el libro Estudios Santiaguistas (León, 1978); y una espléndida monografía, La Banca toscana y la Orden de Santiago, durante el siglo XII, también incluida en aquel libro y cuya primera edición, patrocinada por la Universidad de Valladolid, veria la luz en 1961.

d) La temática judeo-conversa, en la que también Benito Ruano sobresaldría muy pronto como consumado estudioso, dedicándole varios interesantes trabajos igualmente reunidos, en parte, en un volumen publicado en 1976 bajo el título Los origenes del problema converso. 
Con varios libros en su haber, tres decenas, aproximadamente, de artículos, algunos de considerable extensión, e infinidad de reseñas, crónicas y aportaciones bibliográficas que se vinculan en su mayor parte a su actividad como secretario de la revista «Hispania»; con la experiencia acumulada en una larga etapa de docencia en las disciplinas de la Cátedra de Historia General de España de la Facultad de Filosofía y Letras de la Universidad Central y con el refrendo de la sólida formación adquirida al lado de maestros como Montero Díaz o Rumeu de Armas, Eloy Benito Ruano accede en 1964 a la Cátedra universitaria.

Se iniciaba para él una nueva etapa de la que ibamos a ser privilegiados beneficiarios sus nuevos discípulos de la lejana Asturias: quien esto escribe y los compañeros que junto al Profesor Benito Ruano, bajo su dirección y apoyo constantes, somos ahora depositarios del legado científico y académico que dejó en las Universidades hermanas de Oviedo y León el magisterio ejemplar de don Eloy.

Recientemente y con ocasión del libro homenaje que le dedicábamos sus discípulos asturianos, evocaba yo la "etapa ovetense" del Profesor Benito Ruano. Larga etapa que cubre los diecisiete años que median entre 1964, cuando en septiembre se posesiona de la Cátedra de Historia General de España en la Universidad de Oviedo, y septiembre de 1981, fecha de su traslado y anclaje definitivo en el Madrid natal, para hacerse cargo de la Cátedra de Historia Medieval de la UNED que desempeñaría hasta el momento de su reciente jubilación. No voy a insistir ahora en lo ya dicho entonces. Sí señalaré que su dilatada estancia en Asturias, contra lo que solía ser norma en los catedráticos foramontanos que aquí arribaban, constituye, sin duda, el marco central de referencia en la trayectoria científica y académica de don Eloy, su época de plenitud, y a escala personal, y creo que él no dudaria en confirmarlo, la que más hondamente ha marcado su vida.

Para nosotros, los discípulos ovetenses, y englobo en este grupo a quienes, procedentes de la Escuela de Oviedo, formarian, precisamente de la mano de don Eloy, en los cuadros docentes iniciales de la Universidad de León, creada a finales de la década de los setenta, el azar de un destino administrativo nos iba a brindar el don impagable no sólo de recibir su magisterio científico sino, y sobre todo, de ser beneficiarios del ejemplo y de la amistad de quien ha sido siempre, ante todo, un caballero universitario. Porque ésta es, quizá la calificación que mejor define 
el talante personal de don Eloy, en el ejercicio de su noble oficio docente y en su actitud con la legión de compañeros, discípulos y amigos que ha ido cosechando a lo largo de su vida, dentro y fuera de las aulas.

Parafraseando a don Claudio Sánchez-Albornoz, podríamos decir que Benito Ruano recibía en Oviedo «una herencia compleja y difícil». En septiembre de 1964, después de obtener en brillante oposición la Cátedra de Historia General de España de la Facultad de Filosofía y Letras de la Universidad ovetense, llega a la Vetusta clariniana, "Corte en lejano tiempo», el ya exprofesor de la Universidad Central madrileña.

La mudanza de escenario no podía ser mayor. Muchas veces evocaría después Eloy esa su arribada forzosa -o mejor forzada- a las brumosas tierras norteñas, que visitaba por vez primera y que para él habían sido sólo, hasta entonces, un vago punto de referencia en el mapa y en sus propios estudios de medievalista. Como el peregrino Bartolomé de Fontana a principios del siglo XVI, recordaría Eloy, en esas evocaciones, la impresión que le produjo la primera visión de Asturias, celada por la neblina otoñal de los puertos, desde el imponente paso de Pajares, su llegada a Oviedo y la toma de posesión de la flamante credencial de Catedrático en el Rectorado del viejo y venerable caserón fundado por el inquisidor Valdés Salas, que daba cobijo entonces a las facultades de Derecho y Filosofía y Letras.

Muy pronto fue uno más, "uno de los nuestros», como me comentaría luego otro compañero común, refiriéndose al pequeño familiar claustro -iba a escribir clan- que componía el cuadro docente de la Facultad de Letras.

Eloy Benito sucedía en la Cátedra a quien había sido en las aulas, ya en los años postreros de su docencia activa, mi maestro entrañable, gran medievalista y figura central del movimiento cultural asturiano del presente siglo: don Juan Uría Ríu. Y asentado en aquel grato ambiente de una pequeña e histórica Universidad de provincias, Eloy comenzó a hacer lo que había hecho hasta entonces siempre; lo que, en rigor, sólo sabía y podía hacer: trabajar, trabajar con una ilusión y generosidad sin límites. Sin pensar en un probable $y$, en aquellas circunstancias, acaso deseable retorno a la Universidad de procedencia o a otra de mayor lustre, y ocasiones de hacerlo no le iban a faltar. Se entregó plenamente a la misión que el destino le había confiado y que él asumió como propia e irrenunciable: forjar discípulos, crear escuela, en definitiva, servir a la que ha sido durante diecisiete largos y fecundos años y seguirá siendo siempre "su Universidad", la de 
Oviedo. Y a la propia sociedad asturiana, a la que devolvió con creces el cariño y la confianza con que acogió, en 1964, la llegada de aquel caballero venido de lejanas tierras.

La inusual capacidad de trabajo del Profesor Benito Ruano se pone de manifiesto en los diversos cargos de gobierno académico que ocupa y en la dinámica actividad que despliega en esa etapa ovetense. Desempeña sucesivamente la secretaría de la Facultad de Filosofía y Letras (1964) y el Decanato de la misma (1977). En 1965 y bajo su impulso se constituye, dentro de la misma Facultad, la Sección de Historia; y en 1972 se le confía la creación del Colegio Universitario de León, dependiente de la Universidad de Oviedo, en el que iniciarían su carrera docente varios de sus discípulos ovetenses y del que sería director hasta que, en 1980 , se creaba formalmente la nueva Universidad leonesa, de cuya Facultad de Filosofía y Letras sería primer Decano en funciones durante el curso 1980-81.

Durante nueve años, hasta que, en 1973, forma hogar propio en Oviedo, sería, además, director del Colegio Mayor San Gregorio, cuyos colegiales de entonces recuerdan todavía hoy con especial cariño -pude comprobarlo en numerosas ocasiones- a quien fue para ellos, sobre todo, un consejero y amigo leal.

De su labor al frente del Departamento de Historia Medieval de la Universidad de Oviedo, que el creó en 1966, soy testigo de excepción en mi condición de colaborador, desde el primer momento, en aquella ilusionada empresa que hoy tratamos de continuar sus discípulos. Puso en marcha la revista "Asturiensia Medievalia» (1972), en cuya última entrega (1985-1986) le rendiamos cálido y sentido homenaje cuantos nos formamos, en Oviedo y León, bajo su magisterio; y una serie de publicaciones monográficas que, continuada hasta el presente y centrada fundamentalmente en el estudio del Medievo asturiano, recogen una muestra significativa de la labor investigadora realizada bajo su dirección y estímulo por quienes fuimos sus discípulos y colaboradores.

Él mismo, en su densa bibliografía correspondiente a la etapa ovetense, dedicaría no pocos de sus trabajos de entonces a nuestra historia regional: más de una veintena entre los que se cuentan aportaciones de interés tan sobresaliente como su estudio sobre las Hermandades en Asturias durante la Edad Media, que fue discurso de ingreso en el Instituto de Estudios Asturianos (1972); o los capítulos dedicados a La época de la Monarquía asturiana, que cubren la primera parte del t. IV de la 
«Historia de Asturias» (1977 y ss.), obra colectiva de gran empeño que él se encargó de dirigir y coordinar.

Su estrecha vinculación con el Colegio Universitario de León, primero, y desde 1980 con su nueva Universidad, dejaría también honda huella en la labor investigadora del Profesor Benito Ruano, que contabjliza notables aportaciones al conocimiento del Medievo leonés. Asturias y León se suman así a Canarias y Toledo en la parcela de historiografía en marcos regionales que constituye, como ya vimos, uno de los grandes bloques temáticos de la bibliografía de Benito Ruano. A su entusiasta empeño se debe igualmente la formación de la nutrida colección que integran las series de publicaciones, cerca de treinta volúmenes, que verían la luz a partir de 1972 desde el Colegio Universitario de León.

El alejamiento físico de Madrid no impediría a don Eloy en esos años continuar desarrollando una infatigable labor de proyección científica internacionalista, puesta de relieve en su activa presencia en reuniones, congresos y cursos para los que regularmente se recaba su concurso fuera de nuestras fronteras; $y$, sobre todo, actuando de eficaz coordinador de la comunidad de historiadores hispanos, plenamente integrada en las coordenadas de la historiografía mundial, desde sus cargos en la Asociación Española de Ciencias Históricas, de la que ha sido sucesivamente vicesecretario, secretario, vicepresidente $y$, finalmente, presidente desde 1975, participando en todos los Congresos Internacionales de Historia celebrados desde 1955 en diversas sedes: Roma, Estocolmo, Viena, Moscú, San Francisco, Bucarest y Sttutgart.

Numerosas Academias e Instituciones científicas españolas y extranjeras incorporan al Profesor Benito Ruano a sus cuadros, prueba de reconocimiento de su dilatada y valiosa labor historiográfica, de la que da clara idea el repaso a su extensa bibiografía, que continuará incidiendo, fundamentalmente, en los cuatro grandes bloques temáticos que se perfilaban, como ya vimos, en su primera y fecunda etapa de investigador.

Con el comienzo del curso 1981-1982, se abre un nuevo capítulo en la carrera universitaria del Profesor Benito Ruano. Ya como Catedrático de Historia Medieval de España, al darse la opción a los «historiadores generales" de acogerse a la titulación más congruente con su propia especialización científica, accede por concurso de traslado a la Universi- 
dad Nacional de Educación a Distancia. Me tocaba el honor y la responsabilidad de ocupar el lugar desde el que durante tantos años y con tanto fruto había trabajado mi querido maestro en esta Universidad de Oviedo, que sigue siendo para él el hogar académico donde acaso vivió las más gratas experiencias de su ejemplar trayectoria vocacional y profesional.

El mismo entusiasmo y entrega que había prodigado a sus discípulos ovetenses y leoneses lo dispensaría en estos últimos años de docencia activa a sus nuevos colaboradores de la UNED, que le rinden ahora el homenaje debido al maestro común. En septiembre de 1987, en la plenitud de su carrera universitaria y de su actividad científica, le llegaba prematuramente a Eloy Benito Ruano la hora de la jubilación administrativa. Desde entonces y a propuesta del Departamento de Historia Medieval que había dirigido durante los seis últimos años de docencia activa, continúa vinculado a la Universidad Nacional de Educación a Distancia en calidad de Catedrático Emérito.

Con la jubilación se abría ante el Profesor Benito Ruano una nueva y gratísima situación: la derivada de su elección como miembro de número de la Real Academia de la Historia, de la que había sido correspondiente desde 1972, y que suponía el reconocimiento de los muchos méritos acumulados a lo largo de su tensa vida de docente e investigador y de su relevante personalidad científica en el horizonte del actual medievalismo español.

El 22 de mayo de 1988 nos reuníamos en la Academia de la Historia un numerosísimo grupo de discípulos, compañeros y amigos para acompañar a Eloy en el acto, entrañable y solemne, de su investidura académica. Y para escuchar una hermosa lección del maestro - De la alteridad en la Historia - que supone, a través de sus 105 densas y documentadas páginas, un nuevo ejemplo del buen hacer de su autor que sintoniza, en esta espléndida obra, con las más recientes líneas de interés de la historiografía mundial. El Discurso de Eloy Benito Ruano, cuyo contenido glosaría magistralmente el Profesor Rumeu de Armas en la recepción del nuevo académico, constituye una elocuente respuesta, desde el ámbito del medievalismo hispano, a una de las propuestas de grandes temas de investigación que mayor aceptación había tenido en el último de los Congresos Internacionales de Ciencias Históricas hasta ahora celebrados (el XVI, que tuvo lugar en Sttutgart en agosto de 1985): L'image de l'autre.

Sobre el nuevo académico gravita ahora la honrosa responsabilıdad de llevar a término la organización del XVII Congreso Internacional de 
Historia, que tendrá su sede en la capital de España en 1990. La presencia de Eloy Benito Ruano al frente del comité organizador de esta magna asamblea científica es ya toda una garantía de éxito.

$Y$ termino. No han pretendido ser estas deshilvanadas cuartillas, escritas más al dictado del corazón que de la fría y reflexiva exégesis, una semblanza cerrada sobre la vida y obra del maestro. Porque de esa vida ejemplar y en plenitud hay que esperar todavía muchos frutos.

Sin otros títulos que el de ser uno más de sus muchos discípulos y respondiendo al honroso requerimiento que me hacían los compañeros de la Universidad Nacional de Educación a Distancia, no hago más que sumarme al homenaje de gratitud y de cariño que la UNED, llevando la voz de toda la Universidad española, tributa ahora a quien ha sido y es uno de sus más queridos y admirados representantes. 
